\title{
Inhibitory Effects of Genistein on Vascular Smooth Muscle Cell Proliferation Induced by Ox-LDL: Role of BKCa Channels
}

\author{
Bing Bai $(\mathbb{D}$, Nanjuan Lu $(\mathbb{D}$, Wei Zhang $(\mathbb{D}$, Jinghan Lin $(\mathbb{D}$, Tingting Zhao $\mathbb{D}$,

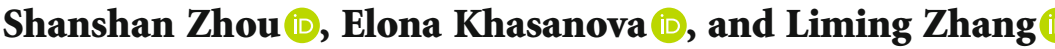 \\ Department of Neurology, The First Affiliated Hospital of Harbin Medical University, 23 Youzheng Street, Harbin, \\ 150001 Heilongjiang Province, China
}

Correspondence should be addressed to Liming Zhang; limingzhanghmu@outlook.com

Received 21 August 2020; Accepted 4 December 2020; Published 14 December 2020

Academic Editor: Syed Ibrahim Rizvi

Copyright ( 2020 Bing Bai et al. This is an open access article distributed under the Creative Commons Attribution License, which permits unrestricted use, distribution, and reproduction in any medium, provided the original work is properly cited.

\begin{abstract}
Background. Oxidized low-density lipoprotein (Ox-LDL) is a crucial pathogenic factor for vascular diseases, which can induce the proliferation of vascular smooth muscle cells (VSMCs). Genistein is the main component of soybean isoflavone. Genistein has a variety of pharmacological properties in the treatment of vascular diseases and a promising clinical application. Large-conductance calcium-activated potassium (BKCa) channels are the primary type of potassium channels in VSMCs, which regulate various biological functions of VSMCs. However, whether genistein exerts an antiproliferation effect on Ox-LDL-stimulated VSMCs remains unclear. The current study is aimed at elucidating the effect of genistein on the Ox-LDL-stimulated proliferation of VSMCs and its possible molecular mechanism, especially the electrophysiological mechanism related to BKCa channels. Methods. Monoculture VSMC was obtained by an acute enzyme-dispersing method. The proliferation of cells was measured by CCK- 8 , cell cycle, and proliferating cell nuclear antigen (PCNA) expression. The BKCa whole-cell currents were measured by patch-clamp. Results. Ox-LDL treatment induced the proliferation of VSMCs, upregulated the BKCa protein expression, and increased the density of BKCa currents, while genistein significantly inhibited these effects caused by Ox-LDL. BKCa channels exerted a regulatory role in the proliferation of VSMCs in response to Ox-LDL. The inhibition of BKCa channels suppressed Ox-LDLstimulated VSMC proliferation, while the activation of BKCa channels showed the opposite effect. Moreover, genistein suppressed the activity of BKCa, including protein expression and current density in a protein tyrosine kinase- (PTK-) dependent manner. Conclusion. This study demonstrated that genistein inhibited the Ox-LDL-mediated proliferation of VSMCs by blocking the cell cycle progression; the possible molecular mechanism may be related to PTK-dependent suppression of BKCa channels. Our results provided novel ideas for the application of genistein in the treatment of vascular diseases and proposed a unique insight into the antiproliferative molecular mechanism of genistein.
\end{abstract}

\section{Introduction}

Atherosclerosis, which is characterized by lipid metabolism disorders, is the leading cause of cardiovascular and cerebrovascular diseases, causing significant mortality worldwide [1]. Vascular smooth muscle cells (VSMCs) are the main cell type in the intimal thickening in atherosclerosis. Recent studies have shown that $70 \%$ of cells in atherosclerotic plaques are VSMC-derived [2, 3]. Although the mechanisms of atherosclerosis are complicated, the unrestrained proliferation of VSMCs is of vital importance $[4,5]$. Therefore, it is of critical importance to study the proliferation of VSMCs in pathological conditions for the treatment of atherosclerosis and the development of antiatherosclerotic drugs.

Large-conductance calcium-activated potassium (BKCa) channels are widely distributed in various mammalian tissues and cells, especially in VSMC [6]. BKCa channels can be activated by intracellular $\mathrm{Ca}^{2+}$ or membrane depolarization, which promotes intracellular $\mathrm{K}^{+}$outward and eventually leads to membrane hyperpolarization [7]. The electrophysiological characteristics of the BKCa channel make it a critical target for VSMC dysfunction. It is widely known that $\mathrm{BKCa}$ 
channels are involved in the regulation of vascular tone, and abnormality of BKCa channels leads to vascular diseases, such as hypertension and atherosclerosis [8-10]. Besides, recent studies revealed that $\mathrm{BKCa}$ channels are involved in the proliferation of various cells $[11,12]$, particularly cancer cells $[13,14]$. Although the function of BKCa channels in the proliferation of VSMCs has not been fully characterized until now, in view of the electrophysiological characteristics of BKCa channels, we hypothesized that BKCa channels may play a regulatory role in the proliferation of VSMCs.

Oxidized low-density lipoprotein (Ox-LDL) is an independent risk factor for atherosclerosis. It can promote the occurrence and development of atherosclerosis by inducing the migration and proliferation of VSMCs $[15,16]$. VSMC dysfunction, particularly the abnormal proliferation caused by Ox-LDL, has become the focus of frontier research on atherosclerosis. Ox-LDL can regulate the activity of various ion channels in different cells. It activated L-type $\mathrm{Ca}^{2+}$ channels in cardiac myocytes and A7r5 smooth muscle-derived cell lines $[17,18]$. In endothelial cells, ox-LDL enhanced Kv1.5 protein expression and increased the open-state probability (Po) of BKCa channels, which is closely related to Ox-LDLmediated endothelial proliferation [19]. Nevertheless, whether BKCa channels are involved in the ox-LDL-mediated proliferation of VSMCs has not been extensively studied.

Genistein is a kind of isoflavone extracted mainly from soybean and widely regarded as a phytoestrogen, with a variety of biological properties, including antiproliferative, antiinflammatory, and antioxidative properties [20-23]. The WHO-CARDIAC and other extensive clinical studies have shown that genistein has a beneficial effect on the prevention and treatment of vascular diseases [24]. Based on multiple clinical trials of genistein with various diseases, genistein shows low oral bioavailability, but it has favorable absorption properties in the intestine [25]. Recent studies have shown that genistein can modulate the function of different ion channels, such as chloride channels, voltage-gated sodium channels, and potassium channels [26, 27]. However, it is still far from clear whether genistein possesses an antiproliferative activity on Ox-LDL-stimulated VSMCs, and a potential molecular mechanism involves the regulation of BKCa channels. The BKCa channel is the main potassium channel with multiple regulatory functions in VSMCs; however, it is unknown whether the antiproliferative mechanism of genistein involves the regulation of BKCa channels.

In this study, we hypothesized that genistein may display an antiproliferative effect on Ox-LDL-induced VSMCs, and its potential molecular mechanism may involve the regulation of BKCa channels. The cell proliferation was measured by CCK-8, cell cycle distribution, and the expression of proliferating cell nuclear antigen (PCNA). The activity of BKCa channels was evaluated using the patch-clamp assay and Western blotting. Our results indicated that genistein exerted an antiproliferative effect on VSMCs infused with Ox-LDL. The molecular mechanisms of this process are probably related to the regulation of $\mathrm{BKCa}$ channels by genistein. Our findings may provide new evidence for the application of genistein in Ox-LDL-mediated VSMC dysfunction and also highlight the regulatory function of BKCa channels.

\section{Materials and Methods}

2.1. Cell Isolation and Culture. The rat VSMCs were derived from male Wistar rats. Briefly, male Wistar rats aged 10 weeks with a weight of $200 \pm 20 \mathrm{~g}$ were euthanized by cervical dislocation. Mesenteric arteries were dissected gently from the abdominal aorta, the fat and connective tissues were carefully separated from the arteries, and the arteries were cut into $1 \mathrm{~mm}$ long cylindrical sections. These sections were incubated in solution containing $1.5 \mathrm{mg} / \mathrm{mL}$ papain, $1.5 \mathrm{mg} / \mathrm{mL}$ dithiothreitol, and $1.5 \mathrm{mg} / \mathrm{mL}$ BSA for $20-25 \mathrm{~min}$ at $37^{\circ} \mathrm{C}$, followed by digestion in $1.0 \mathrm{mg} / \mathrm{mL}$ collagenase $\mathrm{F}, 1.5 \mathrm{mg} / \mathrm{mL}$ hyaluronidase, and $1.5 \mathrm{mg} / \mathrm{mL}$ BSA for 6-10 min. Papain, dithiothreitol, BSA, collagenase F, and hyaluronidase were purchased from Sigma-Aldrich (St. Louis, MO, USA). After digestion, the single smooth muscle cells were dissociated by gentle trituration with a pipette. Cells were maintained in DMEM with the supplementation of $20 \%$ fetal bovine serum (Gibco, USA) and $100 \mathrm{U} / \mathrm{mL}$ streptomycin/penicillin (Gibco, USA).

The protocol was approved by the Animal Care and Welfare Committee of the First Affiliated Hospital of Harbin Medical University. The experimental protocols were conducted in accordance with the National Guidelines for the Care and Use of Laboratory Animals.

2.2. Cell Counting Kit-8 (CCK-8) Assay. VSMCs $\left(5 \times 10^{3}\right.$ cells/well) were plated in 96-well plates and synchronized by serum starving for $24 \mathrm{~h}$, and then, cells were treated with $30 \mu \mathrm{mol} / \mathrm{L}$ of NS1619 (Sigma-Aldrich, USA), $100 \mathrm{nmol} / \mathrm{L}$ of iberiotoxin (IBTX, Sigma-Aldrich, USA), or $50 \mu \mathrm{mol} / \mathrm{L}$ of genistein (Sigma-Aldrich, USA) for $6 \mathrm{~h}$ before stimulation with or without $75 \mu \mathrm{g} / \mathrm{mL}$ Ox-LDL (Yiyuan Biotechnologies, Guangzhou, China) [26]. Cell proliferation was quantified by a CCK-8 kit (Dojindo Molecular Technologies, Inc., Kumamoto, Japan).

2.3. Cell Cycle Assay. After cell growth upon subconfluence, they were synchronized under a serum-free condition for $24 \mathrm{~h}$. The cells were pretreated with $30 \mu \mathrm{mol} / \mathrm{L}$ of NS1619, $100 \mathrm{nmol} / \mathrm{L}$ of IBTX, or $50 \mu \mathrm{mol} / \mathrm{L}$ of genistein for $6 \mathrm{~h}$, followed by coincubation with $75 \mu \mathrm{g} / \mathrm{mL}$ of Ox-LDL for another $72 \mathrm{~h}$. The distribution of the cell cycle was determined by a Cell Cycle Analysis Kit (Beyotime, China) using LSR II flow cytometry (BD Biosciences) and analyzed by ModFit software (Verity Software House, Topsham, ME).

2.4. Western Blot Assay. The cells were rinsed with PBS three times and lysed in RIPA buffer $(50 \mathrm{mM}$ Tris- $\mathrm{HCl} \mathrm{pH} \mathrm{7.2,}$ $150 \mathrm{mM} \mathrm{NaCl}, 1 \%$ NP40, 0.1\% SDS, 0.5\% DOC, $1 \mathrm{mM}$ PMSF, and $25 \mathrm{mM} \mathrm{MgCl}_{2}$ ). The proteins were mixed with $2 \mathrm{x}$ Laemmli sample loading buffer (Bio-Rad, USA) and denatured at $95^{\circ} \mathrm{C}$ for $10 \mathrm{~min}$, then separated using 10\% SDS-PAGE gel and transferred to polyvinylidene difluoride membranes (GE Healthcare Life Sciences, USA). After blocking with 5\% nonfat milk in TBST (0.1\% Tween-20), the membranes were incubated in primary antibody for BKCa (ab9276, $1: 1000$, Abcam, Cambridge, UK) or PCNA (ab29, $1: 1000$, Abcam, Cambridge, UK) at $4^{\circ} \mathrm{C}$ overnight followed by incubation with IRDye 800CW Goat anti-Mouse IgG $(\mathrm{H}+\mathrm{L})$ secondary antibody (926-32210, LICOR, USA) for two hours. The bands were developed by an Odyssey $^{\circledR}$ infrared scanner (LI-COR, Lincoln, USA). The 


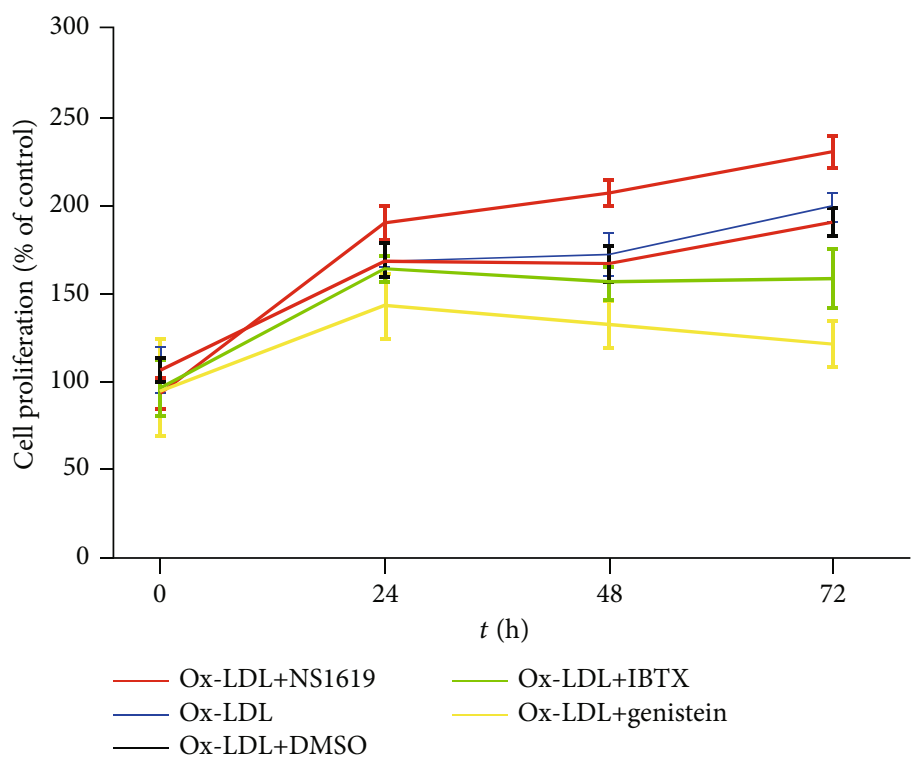

(a)

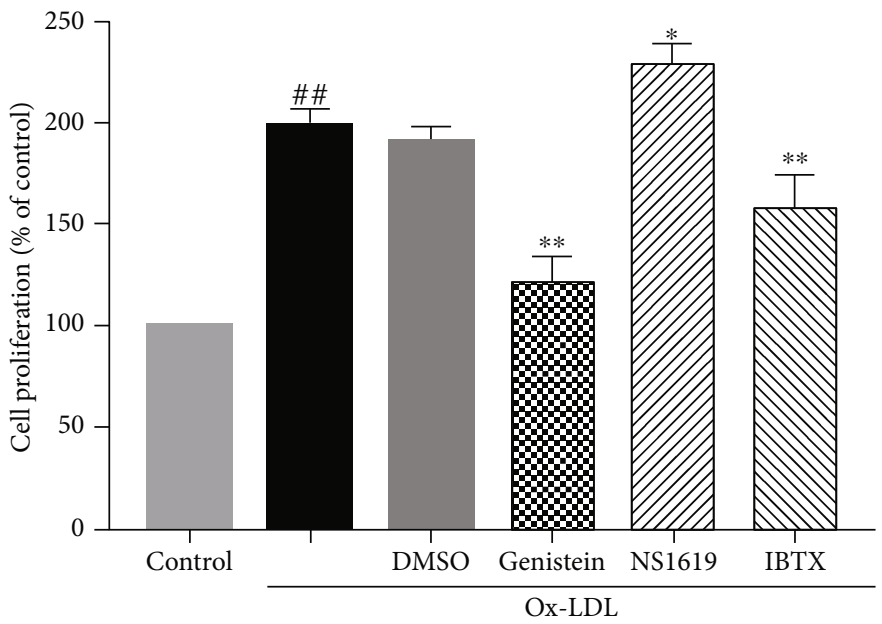

(b)

FIGURE 1: The effect of genistein and BKCa channels on Ox-LDL-induced VSMC proliferation. Cell proliferation was determined by CCK-8. (a) Cell proliferation curves of different treatments. (b) The cell proliferation rate of different groups at $72 \mathrm{~h}$. Ox-LDL increases the proliferation of primary cultured VSMCs. Genistein and IBTX inhibit Ox-LDL-increased proliferation, while NS1619 increases proliferation. DMSO had no significant effect. Results were presented as the mean \pm SD from three independent experiments. ${ }^{\# \#} P<0.01$ vs. control, ${ }^{*} P<0.05$ and ${ }^{* *} P<0.01$ vs. Ox-LDL.

protein expression levels were normalized as the ratio of band intensity to $\beta$-actin (ab6276, 1:5000, Abcam, Cambridge, UK).

2.5. Whole-Cell Patch Recording. Cells were perfused with bath solution containing the following (mM): $\mathrm{NaCl}$ 145.0, $\mathrm{MgCl}_{2}$ 1.2, $\mathrm{CaCl}_{2}$ 1.0, $\mathrm{KCl}$ 5.6, glucose 10.0, and HEPES 10.0. The $\mathrm{pH}$ of the bath solution was adjusted to 7.4. For recording the BKCa currents, $3 \mathrm{mM}$ 4-aminopyridine (Sigma-Aldrich, USA) was used to block the other outward $\mathrm{K}^{+}$currents. The pipette solution consisted of (mM) $\mathrm{KCl} 140.0, \mathrm{CaCl}_{2}$ 0.686, $\mathrm{MgCl}_{2}$ 1.651, EGTA 1.0, $\mathrm{K}_{2}$ ATP 2.0, and HEPES $1.0(\mathrm{pH}$ 7.3). The patch electrode resistance was $4-7 \mathrm{M} \Omega$ after filling with pipette solution. Whole-cell BKCa currents were activated with $500 \mathrm{~ms}$ voltage steps from -40 to $+80 \mathrm{mV}$ in $10 \mathrm{mV}$ increments. The currents were recorded at a holding potential of
$-70 \mathrm{mV}$. After the currents were stabilized, $50 \mu \mathrm{mol} / \mathrm{L}$ genistein, tyrphostin 23, or daidzein (Sigma-Aldrich, USA) was added into the bath solution, and the change of the currents was recorded accordingly. The current density was presented as current amplitude/cell capacitance $(\mathrm{pA} / \mathrm{pF})$. Axopatch $700 \mathrm{~B}$ Amplifier (Axon Instrument, USA), Signal 3.06 software, and CED Power 1401 A/D interface (Cambridge Electronic Design Limited, Cambridge, UK) were used in this study to record whole-cell BKCa currents.

2.6. Statistical Analysis. All experiments were performed in triplicates $(n=3)$, and the values were presented as the mean \pm standard deviation (SD). A comparison between multiple groups was conducted by using one-way ANOVA followed by Bonferroni correction. Student's $t$-tests were used to 


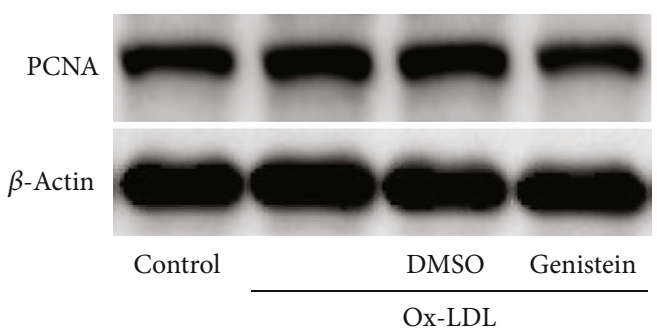

(a)

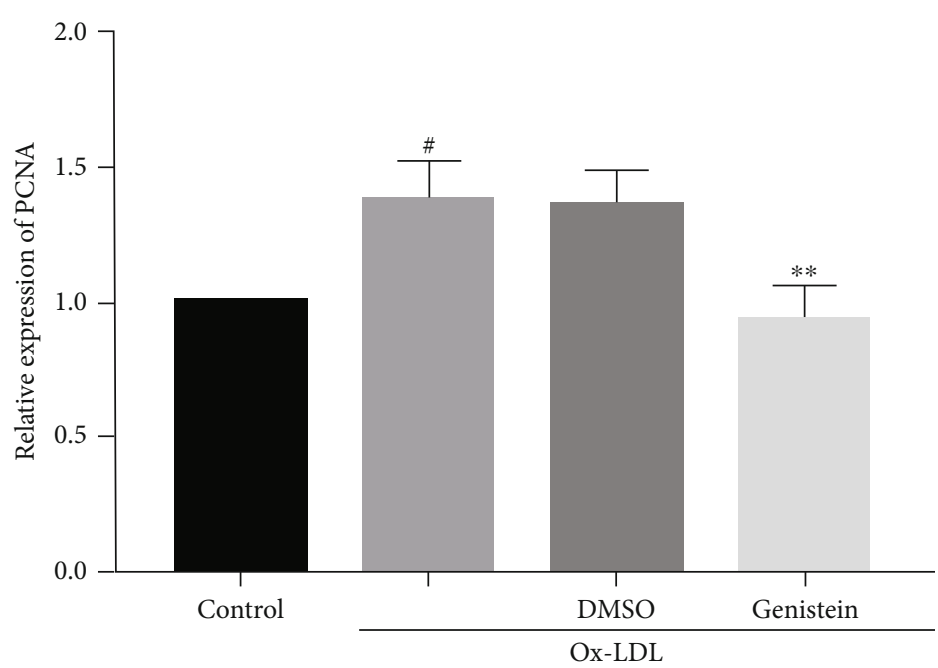

(b)

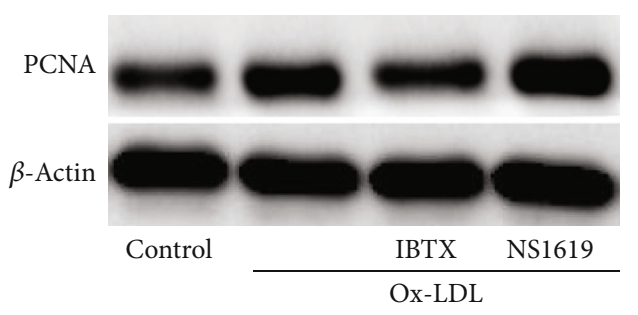

(c)

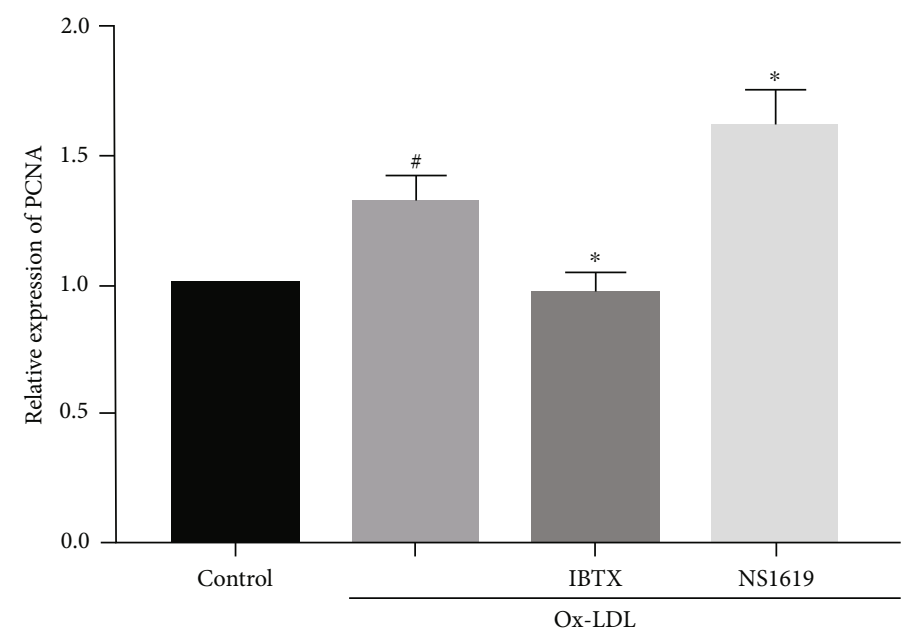

(d)

Figure 2: The effect of genistein and BKCa channels on PCNA expression. (a, b) Ox-LDL enhanced the expression of PCNA while genistein reversed this effect. DMSO had no significant effect. (c, d) The expression of PCNA was upregulated by NS1619 and downregulated by IBTX. ${ }^{\#} P<0.05$ vs. control, ${ }^{*} P<0.05$ and ${ }^{* *} P<0.01$ vs. Ox-LDL. All the results are expressed as the mean \pm SD ( $n=3$ per group).

compare data between two groups. A $P$ value less than 0.05 was considered statistically significant.

\section{Results}

3.1. Effect of Genistein and BKCa on Ox-LDL-Mediated VSMC Proliferation. Initially, the cytotoxicity of NS1619, IBTX, and genistein in VSMCs was determined by a CCK-8 assay. The concentrations of NS1619, IBTX, and genistein at $30 \mu \mathrm{mol} / \mathrm{L}$, $100 \mathrm{nmol} / \mathrm{L}$, and $50 \mu \mathrm{mol} / \mathrm{L}$, respectively, which did not induce cell death were selected for further studies (data not shown). Ox-LDL increased the growth of VSMCs in a time-dependent manner (Figure 1(a)). Figure 1(b) showed the proliferation rate of VSMCs at $72 \mathrm{~h}$. Ox-LDL significantly increased the proliferation of VSMCs $(P<0.01$ vs. control). Genistein leads to a significant decrease in cell proliferation $(P<0.01$ vs. Ox-LDL group). NS1619, the specific BKCa channel activator, increased the proliferation of VSMCs whereas IBTX, the specific BKCa channel blocker, suppressed VSMC proliferation. DMSO had no significant effect on cell proliferation. 

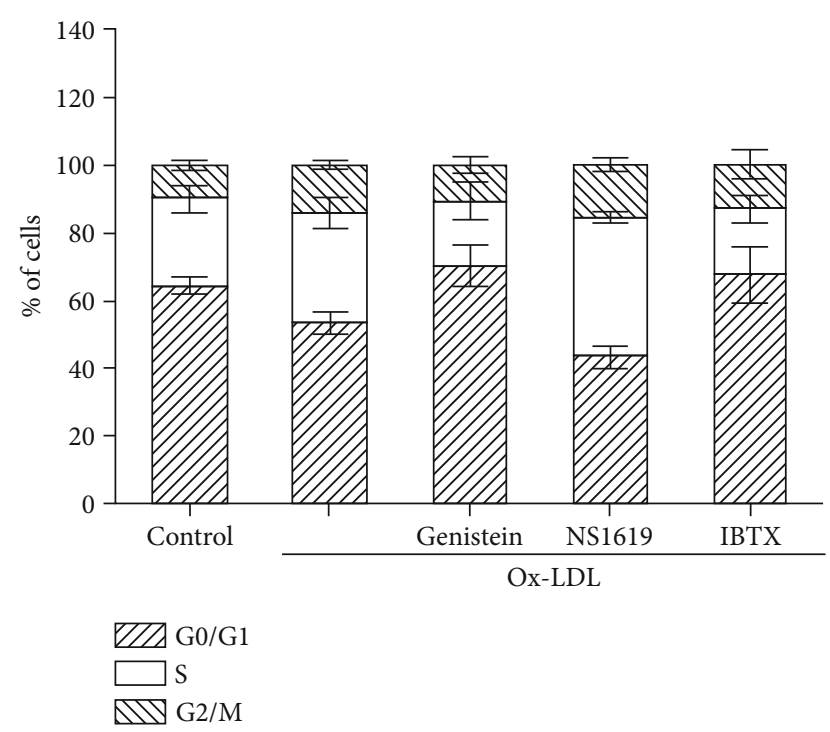

Figure 3: The effect of genistein and BKCa channels on Ox-LDLinduced VSMC cell cycle. Cells were pretreated with genistein, NS1619, or IBTX for $6 \mathrm{~h}$ before coincubated with Ox-LDL for another $72 \mathrm{~h}$. The control group is cells without any treatment. The distribution percentages of cells in each phase were presented as the mean $\pm \mathrm{SD}$ ( $n=3$ per group).

To further evaluate the proliferation of VSMCs, the expression of proliferating cell nuclear antigen (PCNA) was measured (Figure 2). PCNA is a proliferation marker which is synthesized in the early phase of G0/G1 and S. The expression of PCNA upregulated after stimulation with Ox-LDL. Genistein and IBTX downregulated the high expression of PCNA caused by Ox-LDL. NS1619 upregulated the expression of PCNA in Ox-LDL-mediated VSMCs.

3.2. Effect of Genistein and BKCa on Ox-LDL-Mediated Cell Cycle Progression. In order to further confirm that genistein and BKCa are involved in the regulation of cell growth, cell cycle analysis was performed. As shown in Figure 3, the percentage of the G0/G1 phase in the Ox-LDL group decreased from $64 \%$ to $53 \%(P<0.01)$ compared to the control group. Genistein significantly increased the G0/G1 phase to $70 \%(P<0.01)$. NS1619 decreased the G0/G1 phase to $43 \%(P<0.01)$, while IBTX increased the ratio to $67 \%$ $(P<0.05)$. These results further confirmed the modulation role of genistein and $\mathrm{BKCa}$ in the proliferation of VSMCs.

3.3. Genistein Downregulates Ox-LDL-Enhanced Protein Expression and Current Level of BKCa Channels. After isolation by the enzymatic digestion method, VSMCs were incubated in a culture medium containing $75 \mu \mathrm{g} / \mathrm{mL}$ Ox-LDL for $72 \mathrm{~h}$, and then, single cells were obtained for patch-clamp. BKCa currents were recorded before and after perfusion with genistein $(50 \mu \mathrm{mol} / \mathrm{L})$. Figure $4(\mathrm{a})$ shows the representative tracing of whole-cell $\mathrm{BKCa}$ currents. BKCa currents were increased by Ox-LDL treatment. The Ox-LDL-enhanced currents were partly reduced by genistein. The voltagecurrent $(I-V)$ curves are displayed in Figure 4(b). As shown in Figure $4(\mathrm{c})$, at the test potential of $-80 \mathrm{mV}$, BKCa current density of the Ox-LDL group $(92.0 \pm 24.7 \mathrm{pA} / \mathrm{pF}, n=15)$ demonstrated a significant increase compared to the control group $(44.3 \pm 15.9 \mathrm{pA} / \mathrm{pF}, n=13, P<0.01)$. However, after perfusion with genistein, the $\mathrm{Ox}$-LDL-enhanced BKCa current density was decreased to $64.7 \pm 17.0 \mathrm{pA} / \mathrm{pF}(n=17)$. Peak current densities were lower in the presence of genistein compared to the Ox-LDL group $(P<0.05)$. DMSO caused no significant changes in BKCa currents.

Furthermore, we investigated the effect of genistein on the expression of BKCa. Ox-LDL treatment induced an upexpression of BKCa protein $(P<0.01$ vs. control) (Figure $4(\mathrm{~d})$ ), whereas genistein reversed the upregulation caused by $\mathrm{Ox}$ LDL $(P<0.01)$. DMSO caused no significant differences in $\mathrm{BKCa}$ expression. These results suggested that genistein downregulated Ox-LDL-mediated protein expression and current level of BKCa channels.

3.4. Effect of Genistein, Tyrphostin 23, and Daidzein on OxLDL-Enhanced Protein Expression and Current Level of BKCa Channels. VSMCs were cultured with or without $75 \mu \mathrm{g} / \mathrm{mL}$ Ox-LDL for $72 \mathrm{~h}$. Whole-cell BKCa currents were recorded before and after perfusion with genistein $(50 \mu \mathrm{mol} / \mathrm{L})$, tyrphostin $23(50 \mu \mathrm{mol} / \mathrm{L})$, or daidzein $(50 \mu \mathrm{mol} / \mathrm{L})$. Figures $5(\mathrm{a})$ and 5 (b) show the representative BKCa current traces and $I-V$ relationship curves. As shown in Figure 5(c), at the test potential of $-80 \mathrm{mV}, \mathrm{Ox}-\mathrm{LDL}$ increased current density to $92 \pm 25.6$ $\mathrm{pA} / \mathrm{pF}$ ( $n=13, P<0.01$ vs. control); genistein and tyrphostin 23 significantly reduced current density to $64.7 \pm 17.6 \mathrm{pA} / \mathrm{pF}$ ( $n=17, P<0.05$ vs. Ox-LDL group) and $60.5 \pm 22.6 \mathrm{pA} / \mathrm{pF}$ ( $n=13, P<0.05$ vs. Ox-LDL group), respectively. Daidzein altered the current density to $101.5 \pm 31.9 \mathrm{pA} / \mathrm{pF} \quad(n=13)$, which had no significant difference with the Ox-LDL group.

VSMCs were pretreated with genistein $(50 \mu \mathrm{mol} / \mathrm{L})$, tyrphostin $23(50 \mu \mathrm{mol} / \mathrm{L})$, or daidzein $(50 \mu \mathrm{mol} / \mathrm{L})$ for $24 \mathrm{~h}$ and coincubated with Ox-LDL for another $72 \mathrm{~h}$. As shown in Figures 5(d) and 5(e), genistein and tyrphostin 23 significantly inhibited Ox-LDL-mediated BKCa expression $(P<0.05)$, while daidzein did not affect BKCa expression. Taken together, these results suggested that genistein and tyrphostin 23 rather than daidzein downregulated Ox-LDLmediated protein expression and current level of $\mathrm{BKCa}$ channels.

3.5. PTK-Dependent Effects of Genistein on BKCa Currents and Protein Expression. Cells were pretreated with or without $50 \mathrm{mmol} / \mathrm{L}$ sodium orthovanadate (SOV) (a protein tyrosine phosphatase inhibitor) for $20 \mathrm{~min}$, and whole-cell $\mathrm{BKCa}$ currents were recorded before and after perfusion with genistein $(50 \mu \mathrm{mol} / \mathrm{L})$. Figures $6(\mathrm{a})$ and $6(\mathrm{~b})$ displayed the representative $\mathrm{BKCa}$ current traces and $I-V$ relationship curves. As shown in Figure 6(c), at the test potential of $-80 \mathrm{mV}$, the $\mathrm{BKCa}$ current density in the Ox-LDL group was $101.1 \pm$ $41.1 \mathrm{pA} / \mathrm{pF} \quad(n=15)$; genistein significantly reduced the currents to $67.1 \pm 15.7 \mathrm{pA} / \mathrm{pF}(n=11, P<0.05)$, while pretreatment with $\mathrm{SOV}$ reversed the currents to $96.8 \pm 39.0$ $\mathrm{pA} / \mathrm{pF}(n=8, P<0.05)$.

Cells were pretreated with or without SOV $(50 \mathrm{mmol} / \mathrm{L})$ for $24 \mathrm{~h}$ before coincubation with genistein $(50 \mu \mathrm{mol} / \mathrm{L})$ for another $72 \mathrm{~h}$. Figures $6(\mathrm{~d})$ and $6(\mathrm{e})$ demonstrated that 


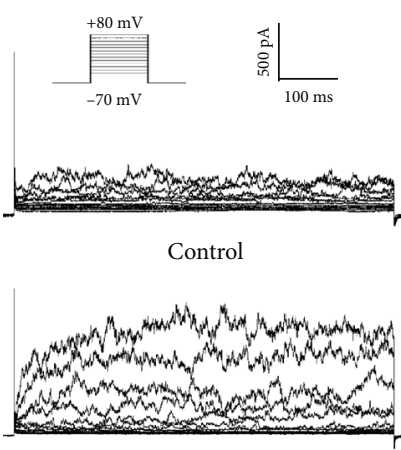

Ox-LDL

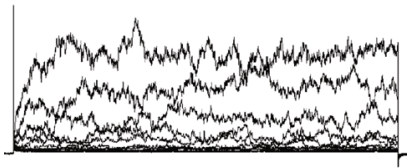

Ox-LDL+DMSO

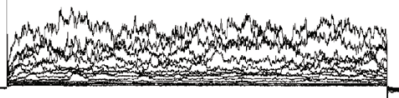

Ox-LDL+genistein

(a)

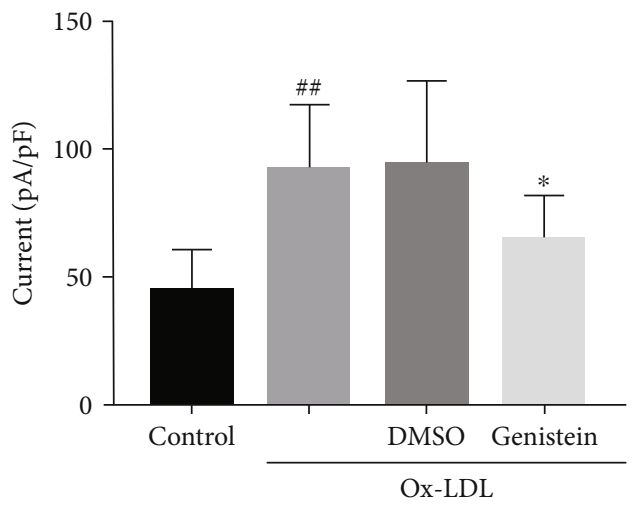

(c)

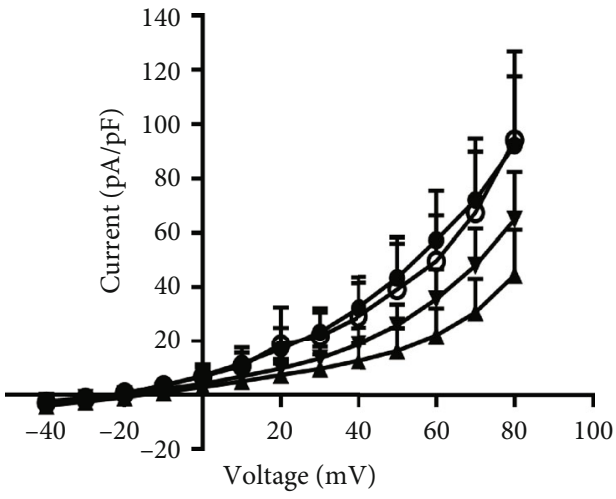

- Ox-LDL $\rightarrow-\mathrm{Ox}-\mathrm{LDL}+\mathrm{G}$

$\odot$ Ox-LDL+DMSO $\quad \longrightarrow$ Control (b)

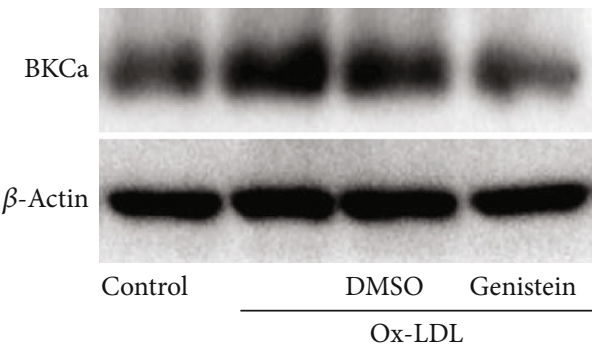

(d)

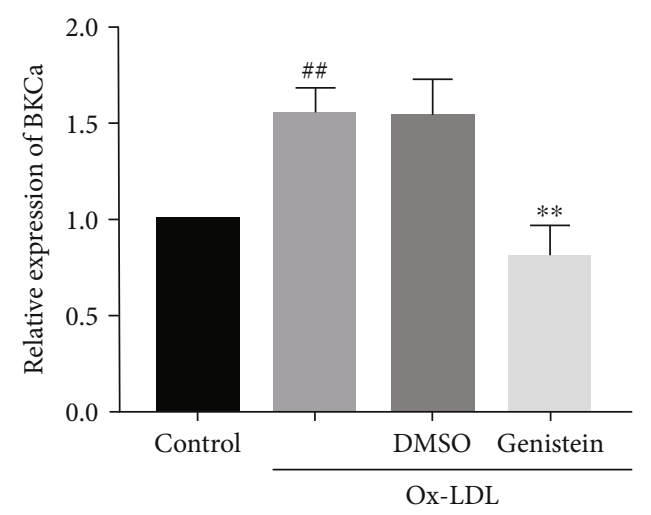

(e)

FIGURE 4: Effect of genistein on protein expression and current level of BKCa channels. (a) Representative BKCa currents. (b) $I$ - $V$ curves of BKCa currents. (c) BKCa peak current densities at the test potential of $-80 \mathrm{mV}$. ${ }^{\# \#} P<0.01$ vs. control, ${ }^{*} P<0.05$ vs. Ox-LDL. (d) Western blot analysis and (e) statistical analysis of BKCa channels. ${ }^{\# \#} P<0.01$ vs. control, ${ }^{* *} P<0.01$ vs. Ox-LDL. All the results are expressed as the mean $\pm \mathrm{SD}(n=3)$. The data indicated that genistein significantly reversed Ox-LDL-enhanced protein expression and current level of BKCa channels. 


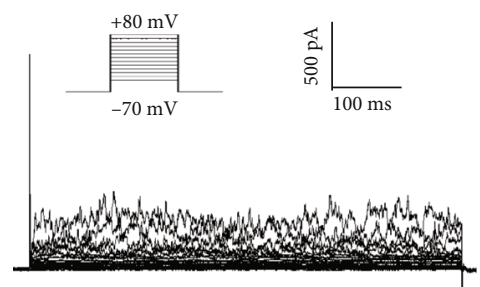

Control

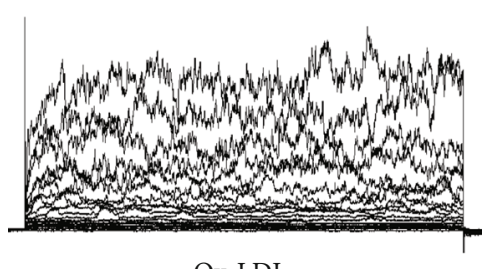

Ox-LDL
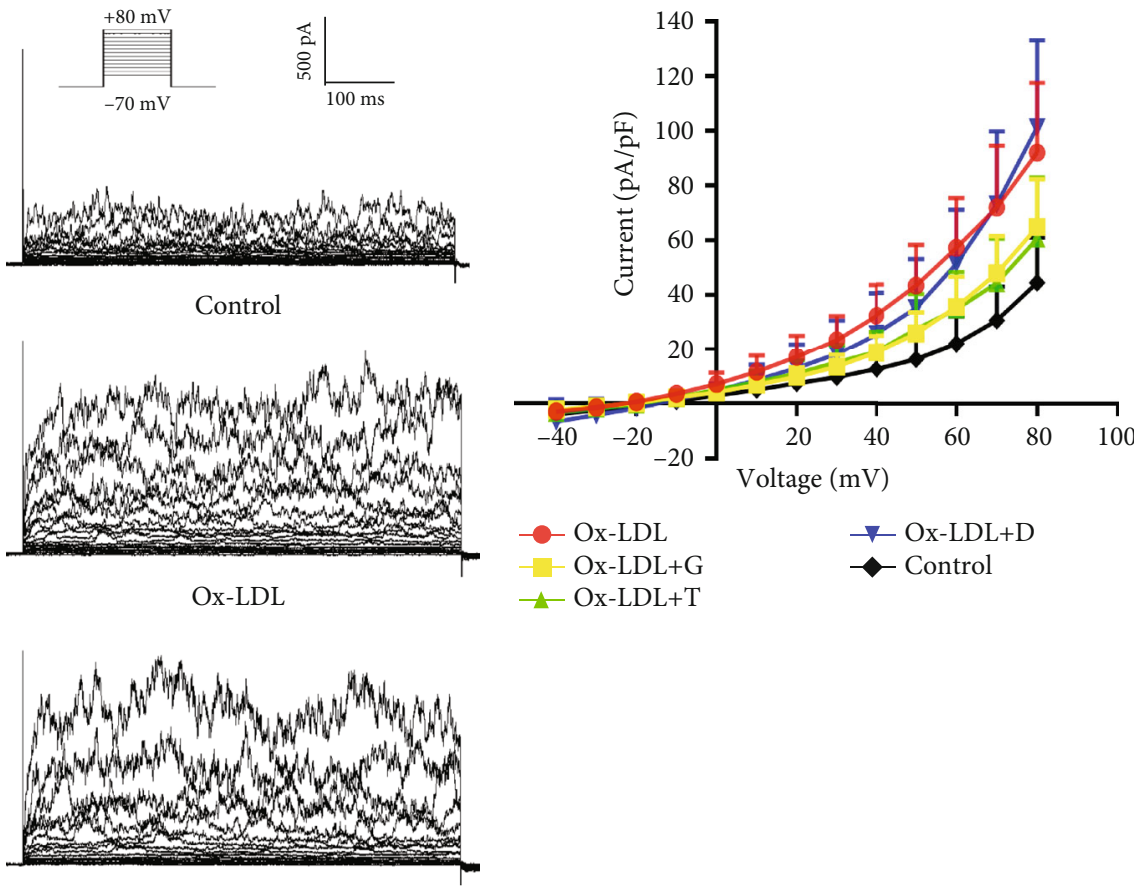

Ox-LDL+D

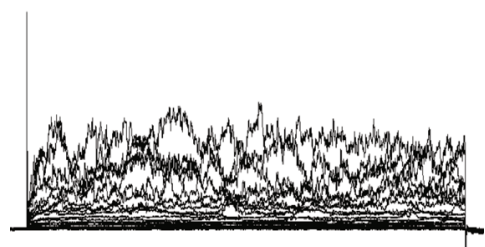

Ox-LDL+G

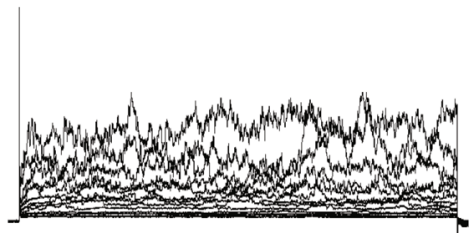

Ox-LDL+T

(a)

(b)

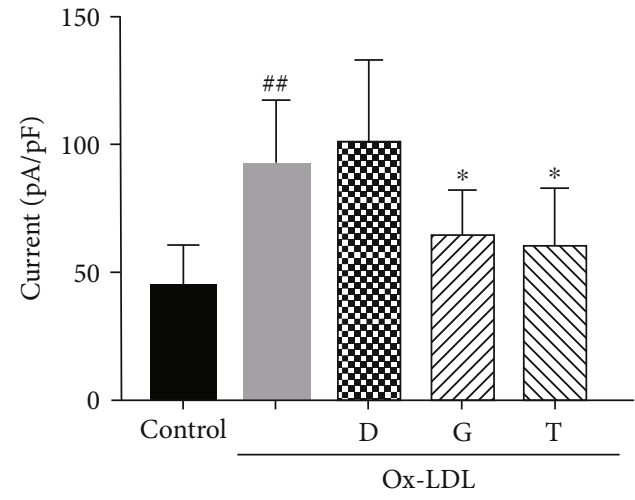

(c)

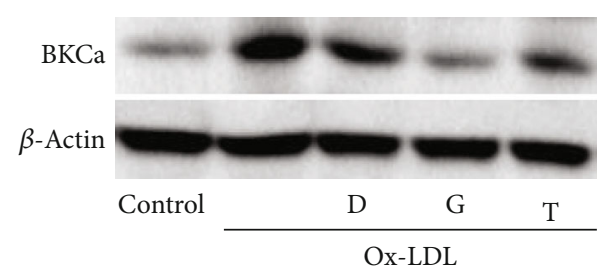

(d)

Figure 5: Continued. 


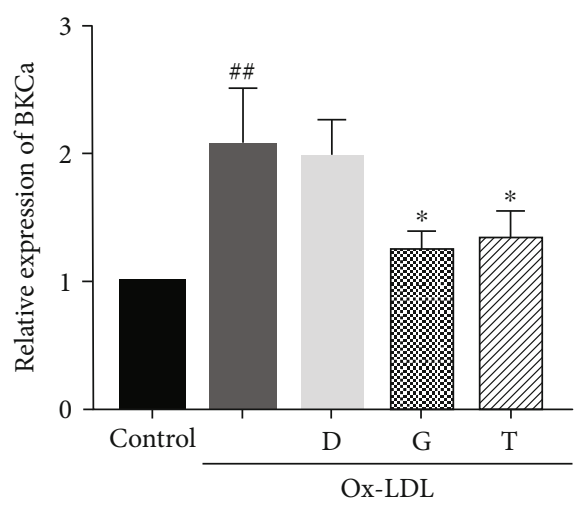

(e)

FIGURE 5: Effect of genistein, tyrphostin 23, and daidzein on protein expression and current level of BKCa channels. (a) Representative BKCa currents. (b) $I-V$ curves of BKCa currents. (c) BKCa peak current densities at the test potential of $-80 \mathrm{mV}$. The results indicated that perfusion with genistein and tyrphostin 23 reversed Ox-LDL-enhanced BKCa currents. ${ }^{\# \#} P<0.01$ vs. control, ${ }^{*} P<0.05$ vs. Ox-LDL. (d) Western blot analysis and (e) statistical analysis of BKCa channels. The results indicated that genistein and tyrphostin 23 reversed Ox-LDL-enhanced BKCa protein expression. ${ }^{\# \#} P<0.01$ vs. control, ${ }^{*} P<0.05$ vs. Ox-LDL. All values are expressed as the mean \pm SD $(n=3)$. Daidzein had no significant effect on protein expression and current level of BKCa channels. Abbreviations: D: daidzein; G: genistein; T: tyrphostin 23.

genistein inhibited Ox-LDL-induced BKCa expression; pretreatment with SOV can reverse the inhibition caused by genistein. These results showed that the inhibition of genistein on BKCa currents and protein expression was related to PTK inhibition.

\section{Discussion}

Genistein is the main component of soybean isoflavone and has multiple pharmacological properties in atherosclerosis prevention [20]. Ox-LDL is involved in almost all pathological stages of atherosclerosis. The abnormal proliferation of VSMCs is a critical pathogenic mechanism of atherosclerosis. In recent years, it has been confirmed that ion channels are closely related to cell proliferation. For instance, CaV 2.1 regulates the proliferation of neural cell [28], and Kv1.3 modulates the proliferation of human VSMC [29]. The BKCa channel is one of the most dominant ion channels in VSMCs and participants in various physiological activities of VSMCs [8]. This study is aimed at determining whether genistein involves in the process of Ox-LDL-mediated VSMC proliferation and revealing the possible role of BKCa channels in this process. Our findings demonstrated that genistein has an antiproliferation effect on Ox-LDL-stimulated VSMCs by inhibition of the protein expression and current density of BKCa channels. These results are of vital importance to the clinical application of genistein for atherosclerosis therapy.

To determine whether the function of BKCa channels is involved in VSMC proliferation in response to Ox-LDL, we utilized the specific channel opener (NS1619) and blocker (IBTX) to regulate the activity of BKCa channels. NS1619 is a selective and reversible activator of BKCa channels and has the potential to modulate cell excitability in neurons and smooth muscle. IBTX is the selective and reversible inhibitor of BKCa channels by binding to a site in the external vestibule of the pore-forming $\alpha$-subunit, whereas NS1619 is the selective agonist to activate the BKCa channel by a direct effect on the $\mathrm{Ca}+$ binding site at the $\mathrm{C}$-terminal end of the $\alpha$-subunit or by the indirect strengthening of the interaction of $\beta$ - to the $\alpha$-binding site [30]. We found that IBTX inhibited the Ox-LDL-mediated VSMC proliferation while NS1619 promoted cell proliferation. Moreover, IBTX induced cell cycle arrest, while NS1619 promoted the course of the cell cycle. These data suggested that the inhibition of BKCa channels reduced Ox-LDL-stimulated VSMC proliferation by preventing cell cycle progression. Our data were consistent with previous reports; inhibition of BKCa channels suppresses endometrial cancer cell proliferation [31]. Previous studies also demonstrated that the inhibition of BKCa channels blocked human bone marrow-derived mesenchymal stem cells in the G0/G1 phase and ultimately suppressed cell growth [32]. Taken together, our results demonstrated that Ox-LDL enhanced the activity of BKCa channels in VSMCs. Moreover, BKCa channels modulated Ox-LDL-induced cell proliferation, indicating that $\mathrm{BKCa}$ channels may serve as a potential target for the treatment of VSMC dysfunction.

Genistein inhibited Ox-LDL-enhanced VSMC proliferation by arresting the cell cycle at the G0/G1 phase. Previous studies on the mechanism of genistein inhibition of cell proliferation mainly focused on kinases and transcription factors. Farruggio et al. reported that genistein regulates $\mathrm{H} 9 \mathrm{C} 2$ proliferation through the modulation of the Akt, ERK1/2, and p38MAPK signaling pathways [33]. Venza et al. reported that genistein inhibits the proliferation of melanoma cells by preventing PGE2-mediated IL-8 expression [34]. Different from previous studies, our study is aimed at investigating whether BKCa channels participate in the mechanism of genistein inhibition of cell proliferation. Our results indicated that genistein not only reduced the density of BKCa currents but also suppressed the expression of the BKCa protein. As previously described, the inhibition of $\mathrm{BKCa}$ channels prevents the abnormal proliferation of VSMCs in response to Ox-LDL. Hence, it is reasonable to ascribe the mechanism of genistein on Ox-LDLmediated VSMC proliferation, at least partially, to its modulation of BKCa channels. 

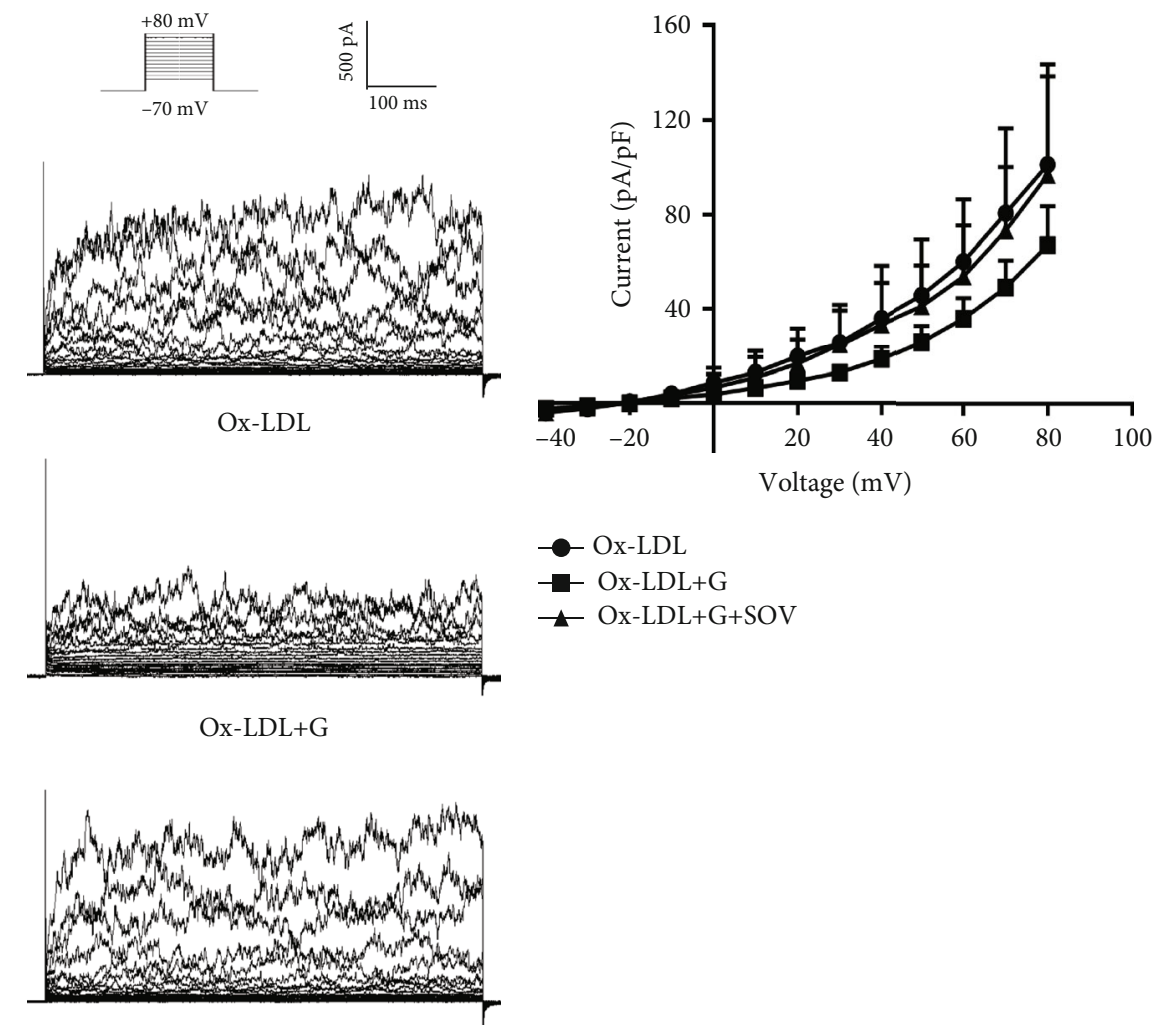

Ox-LDL+G+SOV

(a)

(b)

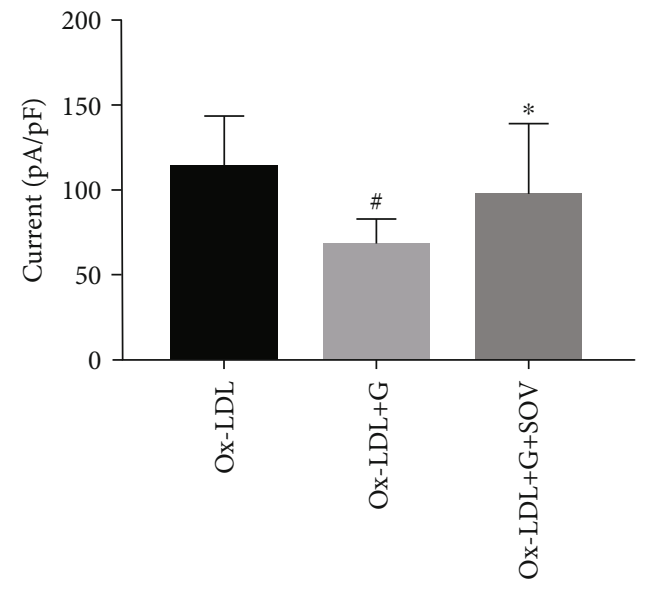

(c)

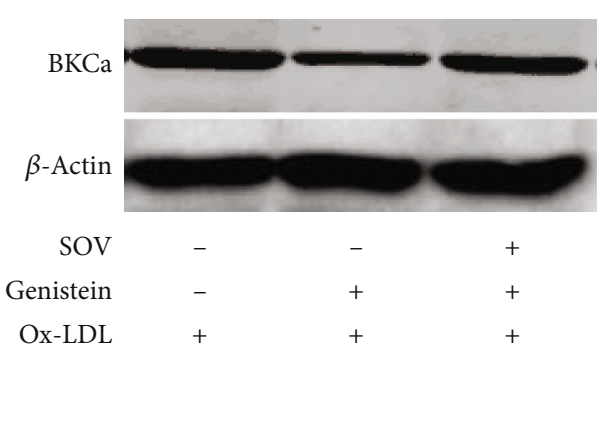

(d)

Figure 6: Continued. 


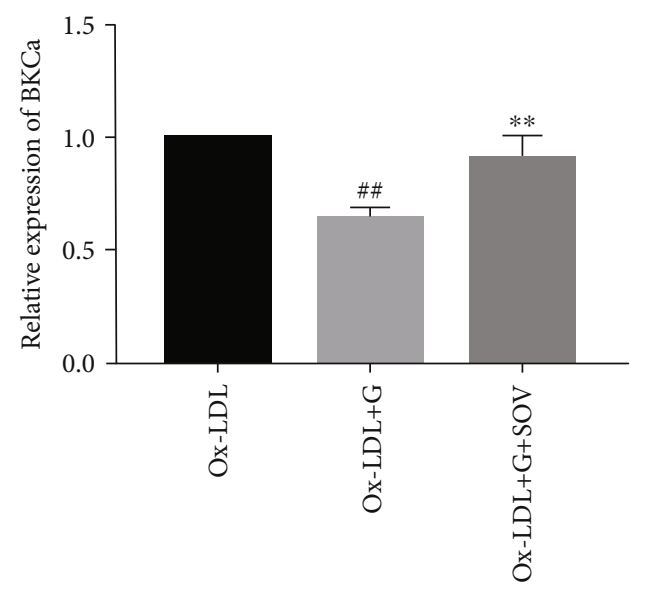

(e)

FIGURE 6: Effects of genistein on BKCa currents and protein expression after preincubation with SOV. (a) Representative BKCa currents recorded in each group. (b) $I-V$ curves of BKCa currents. (c) BKCa peak current densities at the test potential of $-80 \mathrm{mV}$. The data indicated that genistein inhibits Ox-LDL-mediated BKCa currents. The inhibitory effects of genistein were reversed by SOV. ${ }^{*} P<0.05$ vs. Ox-LDL group. (d) Western blot analysis and (e) statistical analysis of BKCa channels. The result indicated that genistein inhibits the expression of BKCa channels. The inhibitory effects of genistein were reversed by SOV. ${ }^{\# \#} P<0.01$ vs. Ox-LDL, ${ }^{* *} P<0.01$ vs. Ox-LDL+G. All the results are expressed as the mean $\pm \mathrm{SD}(n=3)$. Abbreviations: G: genistein; SOV: sodium orthovanadate.

Genistein, as a phytoestrogen and PTK inhibitor, can affect the activity of various ion channels $[35,36]$, such as voltage-gated potassium channels (Kv2.1), voltage-gated sodium channels (Nav), and volume-sensitive chloride channels (Cl.vol) [27, 36, 37]. Nevertheless, the effect of genistein on BKCa channels as a phytoestrogen or whether it is PTK-dependent remains unclear. In this study, daidzein is a structural analogue of genistein, and tyrphostin 23 is widely known as another PTK inhibitor. Our research found that genistein and tyrphostin 23 rather than daidzein inhibited Ox-LDL-enhanced BKCa activity, indicating that the two kinds of chemically distinct PTK inhibition factors can regulate the function of BKCa channels. Genistein and daidzein have a similar benzene ring structure, they are the significant isoflavones extracted from soybean, and both function as a phytoestrogen, but daidzein does not demonstrate bioactivity as a PTK inhibitor [38]. In this research, daidzein did not affect BKCa activity, indirectly suggesting the inhibition of BKCa by genistein is associated with its PTK-dependent bioactivity rather than its function as a phytoestrogen. In addition, after pretreatment with sodium orthovanadate (SOV), a protein tyrosine phosphatase inhibitor, the inhibition of BKCa by genistein and tyrphostin 23 was antagonized, which further confirmed that the inhibition of BKCa channels by genistein was PTK-dependent. Our data are consistent with previous studies [39, 40]. Others have shown that PTKs can modulate several ion channels, such as Kir2.1 channels [41], ROMK channels [42], $\mathrm{K}_{\text {АTP }}$ channels [43], and L-type calcium channels [44]. However, the present research provided the first evidence that genistein suppressed Ox-LDL-enhanced BKCa activity, including protein expression and current density, in primary cultured VSMCs through a PTK-dependent mechanism.

The potential limitation of this study is that our conclusions were based on the research of primary cultured VSMCs, which may not reflect the processes of an intact body. Future investigations are now conducing to validate the effect of genistein on the prevention and treatment of vascular diseases using animal models and in vivo.

In summary, the major findings in our study illustrated that (a) Ox-LDL increased the proliferation of primary cultured VSMCs; (b) genistein induced cell cycle arrest at the G0/G1 phase, which suppressed the abnormal proliferation of VSMCs in response to Ox-LDL; (c) the function of BKCa channels can affect the proliferation of VSMCs; and (d) genistein suppressed the activity of BKCa channels, including protein expression and current density, in a PTK-dependent manner. To the best of our knowledge, our study revealed the functional linkage between genistein and BKCa channels in the proliferation of Ox-LDL-stimulated VSMCs for the first time, provided valuable knowledge about the antiproliferative molecular mechanism of genistein, and emphasized the PTKdependent regulatory mechanisms of genistein on BKCa channels. Nevertheless, future investigations are needed to validate the effect of genistein on the prevention and treatment of vascular diseases using animal models and in vivo.

\section{Conclusions}

In summary, genistein inhibited the function of BKCa channels in a PTK-dependent manner by suppressing cell cycle progression and ultimately reducing Ox-LDL-mediated proliferation of VSMCs. Our findings expanded the knowledge regarding the role of genistein in VSMC dysfunction and provided novel insights into the antiproliferative molecular mechanism of genistein in vascular diseases.

\section{Data Availability}

The data used to support the findings of this study are included in the article. 


\section{Conflicts of Interest}

The authors have no conflicts of interest to declare.

\section{Acknowledgments}

This work was supported by the National Natural Science Foundation of China (Grant no. 81471205) and the Postgraduate Research Innovation Project of Harbin Medical University of China (Grant no. YJSKYCX2018-34HYD).

\section{References}

[1] W. Herrington, B. Lacey, P. Sherliker, J. Armitage, and S. Lewington, "Epidemiology of atherosclerosis and the potential to reduce the global burden of atherothrombotic disease," Circulation Research, vol. 118, no. 4, pp. 535-546, 2016.

[2] S. Allahverdian, A. C. Chehroudi, B. M. McManus, T. Abraham, and G. A. Francis, "Contribution of intimal smooth muscle cells to cholesterol accumulation and macrophage-like cells in human atherosclerosis," Circulation, vol. 129, no. 15, pp. 1551-1559, 2014.

[3] L. S. Shankman, D. Gomez, O. A. Cherepanova et al., "KLF4dependent phenotypic modulation of smooth muscle cells has a key role in atherosclerotic plaque pathogenesis," Nature Medicine, vol. 21, no. 6, pp. 628-637, 2015.

[4] R. Ross and J. A. Glomset, "The pathogenesis of atherosclerosis (first of two parts)," The New England Journal of Medicine, vol. 295, no. 7, pp. 369-377, 1976.

[5] R. Ross and J. A. Glomset, "The pathogenesis of atherosclerosis (second of two parts)," The New England Journal of Medicine, vol. 295, no. 8, pp. 420-425, 1976.

[6] R. Brenner, G. J. Peréz, A. D. Bonev et al., "Vasoregulation by the $\beta 1$ subunit of the calcium-activated potassium channel," Nature, vol. 407, no. 6806, pp. 870-876, 2000.

[7] R. Latorre and S. Brauchi, "Large conductance Ca2+-activated $\mathrm{K}+(\mathrm{BK})$ channel: activation by $\mathrm{Ca} 2+$ and voltage," Biological Research, vol. 39, no. 3, pp. 385-401, 2006.

[8] N. R. Tykocki, E. M. Boerman, and W. F. Jackson, "Smooth muscle ion channels and regulation of vascular tone in resistance arteries and arterioles," Comprehensive Physiology, vol. 7, no. 2, pp. 485-581, 2017.

[9] M. Baranowska, H. Kozłowska, and A. Korbut, "Potassium channels in blood vessels: their role in health and disease," Postępy Higieny i Medycyny Doświadczalnej (Online), vol. 61, pp. 596-605, 2007.

[10] J. Wiecha, B. Schläger, R. Voisard, A. Hannekum, T. Mattfeldt, and V. Hombach, " $\mathrm{Ca}(2+)$-activated $\mathrm{K}+$ channels in human smooth muscle cells of coronary atherosclerotic plaques and coronary media segments," Basic Research in Cardiology, vol. 92, no. 4, pp. 233-239, 1997.

[11] W. F. Jackson, "Potassium channels in regulation of vascular smooth muscle contraction and growth," Advances in Pharmacology, vol. 78, pp. 89-144, 2017.

[12] O. Ayad, C. Magaud, S. Sebille et al., "Functional BKCa channel in human resident cardiac stem cells expressing W8B2," The FEBS Journal, vol. 285, no. 3, pp. 518-530, 2017.

[13] A. Mound, L. Rodat-Despoix, S. Bougarn, H. Ouadid-Ahidouch, and F. Matifat, "Molecular interaction and functional coupling between type 3 inositol 1,4,5-trisphosphate receptor and $\mathrm{BK}_{\mathrm{Ca}}$ channel stimulate breast cancer cell proliferation,"
European Journal of Cancer, vol. 49, no. 17, pp. 3738-3751, 2013.

[14] M. Bury, A. Girault, V. Mégalizzi et al., “Ophiobolin A induces paraptosis-like cell death in human glioblastoma cells by decreasing BKCa channel activity," Cell Death \& Disease, vol. 4, no. 3, article e561, 2013.

[15] A. Trpkovic, I. Resanovic, J. Stanimirovic et al., "Oxidized lowdensity lipoprotein as a biomarker of cardiovascular diseases," Critical Reviews in Clinical Laboratory Sciences, vol. 52, no. 2, pp. 70-85, 2015.

[16] J. Liu, Y. Ren, L. Kang, and L. Zhang, "Oxidized low-density lipoprotein increases the proliferation and migration of human coronary artery smooth muscle cells through the upregulation of osteopontin," International Journal of Molecular Medicine, vol. 33, no. 5, pp. 1341-1347, 2014.

[17] Y. Hammoud, T. Rice, and J. J. Mackrill, "Oxysterols modulate calcium signalling in the A7r5 aortic smooth muscle cell- line," Biochimie, vol. 95, no. 3, pp. 568-577, 2013.

[18] I. M. Fearon, "OxLDL enhances L-type Ca2+ currents via lysophosphatidylcholine-induced mitochondrial reactive oxygen species (ROS) production," Cardiovascular Research, vol. 69, no. 4, pp. 855-864, 2006.

[19] C. R. Kuhlmann, M. Schäfer, F. Li et al., "Modulation of endothelial $\mathrm{Ca}(2+)$-activated $\mathrm{K}(+)$ channels by oxidized LDL and its contribution to endothelial proliferation," Cardiovascular Research, vol. 60, no. 3, pp. 626-634, 2003.

[20] V. Mukund, D. Mukund, V. Sharma, M. Mannarapu, and A. Alam, "Genistein: its role in metabolic diseases and cancer," Critical Reviews in Oncology/Hematology, vol. 119, pp. 13-22, 2017.

[21] K. Polkowski, J. Popiolkiewicz, P. Krzeczynski et al., "Cytostatic and cytotoxic activity of synthetic genistein glycosides against human cancer cell lines," Cancer Letters, vol. 203, no. 1, pp. 59-69, 2004.

[22] A. A. Ganai and H. Farooqi, "Bioactivity of genistein: a review of in vitro and in vivo studies," Biomedicine \& Pharmacotherapy, vol. 76, pp. 30-38, 2015.

[23] H. Zhang, Z. Zhao, X. Pang et al., "Genistein protects against oxLDL-induced inflammation through microRNA-155/SOCS1mediated repression of NF-kB signaling pathway in HUVECs," Inflammation, vol. 40, no. 4, pp. 1450-1459, 2017.

[24] E. H. Moriguchi, Y. Moriguchi, and Y. Yamori, "Impact of diet on the cardiovascular risk profile of Japanese immigrants living in Brazil: contributions of World Health Organization CARDIAC and MONALISA studies," Clinical and Experimental Pharmacology \& Physiology, vol. 31, Suppl 2, pp. S5-S7, 2004.

[25] Z. Yang, K. Kulkarni, W. Zhu, and M. Hu, "Bioavailability and pharmacokinetics of genistein: mechanistic studies on its ADME," Anti-Cancer Agents in Medicinal Chemistry, vol. 12, no. 10, pp. 1264-1280, 2012.

[26] P. J. French, J. Bijman, A. G. Bot, W. E. Boomaars, B. J. Scholte, and H. R. de Jonge, "Genistein activates CFTR Cl- channels via a tyrosine kinase- and protein phosphatase-independent mechanism," The American Journal of Physiology, vol. 273, no. 2, pp. C747-C753, 1997.

[27] Y. X. Wang, Z. H. Xia, X. Jiang et al., "Genistein inhibits A $325-35$-induced neuronal death with changes in the electrophysiological properties of voltage-gated sodium and potassium channels," Cellular and Molecular Neurobiology, vol. 39, no. 6, pp. 809-822, 2019. 
[28] F. Nigussie, P. S. Huang, K. Lukauskis, B. Bawa, E. Moussa, and L. C. Abbott, "Neural cell proliferation and survival in the hippocampus of adult CaV 2.1 calcium ion channel mutant mice," Brain Research, vol. 1650, pp. 162-171, 2016.

[29] P. Cidad, E. Miguel-Velado, C. Ruiz-McDavitt, et al., "Kv1.3 channels modulate human vascular smooth muscle cells proliferation independently of mTOR signaling pathway," Pflügers Archiv, vol. 467, no. 8, pp. 1711-1722, 2015.

[30] S. Ghatta, D. Nimmagadda, X. Xu, and S. T. O'Rourke, "Largeconductance, calcium-activated potassium channels: structural and functional implications," Pharmacology \& Therapeutics, vol. 110, no. 1, pp. 103-116, 2006.

[31] N. Li, L. Liu, G. Li, M. Xia, C. Du, and Z. Zheng, "The role of $\mathrm{BKCa}$ in endometrial cancer HEC-1-B cell proliferation and migration," Gene, vol. 655, pp. 42-47, 2018.

[32] Y. Y. Zhang, J. Yue, H. Che, H. Y. Sun, H. F. Tse, and G. R. Li, "BKCa and hEag1 channels regulate cell proliferation and differentiation in human bone marrow-derived mesenchymal stem cells," Journal of Cellular Physiology, vol. 229, no. 2, pp. 202-212, 2014.

[33] S. Farruggio, G. Raina, G. Cocomazzi et al., "Genistein improves viability, proliferation and mitochondrial function of cardiomyoblasts cultured in physiologic and peroxidative conditions," International Journal of Molecular Medicine, vol. 44, no. 6, pp. 2298-2310, 2019.

[34] I. Venza, M. Visalli, R. Oteri, C. Beninati, D. Teti, and M. Venza, "Genistein reduces proliferation of EP3-expressing melanoma cells through inhibition of PGE2-induced IL-8 expression," International Immunopharmacology, vol. 62, pp. 86-95, 2018.

[35] T. Akiyama, J. Ishida, S. Nakagawa et al., "Genistein, a specific inhibitor of tyrosine-specific protein kinases," The Journal of Biological Chemistry, vol. 262, no. 12, pp. 5592-5595, 1987.

[36] I. A. Aréchiga-Figueroa, R. Morán-Zendejas, M. DelgadoRamírez, and A. A. Rodríguez-Menchaca, "Phytochemicals genistein and capsaicin modulate Kv2.1 channel gating," Pharmacological Reports, vol. 69, no. 6, pp. 1145-1153, 2017.

[37] X. L. Du, Z. Gao, C. P. Lau et al., "Differential effects of tyrosine kinase inhibitors on volume-sensitive chloride current in human atrial myocytes," The Journal of General Physiology, vol. 123, no. 4, pp. 427-439, 2004.

[38] L. Křížová, K. Dadáková, J. Kašparovská, and T. Kašparovský, "Isoflavones," Molecules, vol. 24, no. 6, p. 1076, 2019.

[39] X. Wang, T. Zhao, S. Zhou, L. Sun, L. Zhang, and G. Yu, " $\mathrm{Mg}^{2+}$-dependent modulation of BKCachannels by genistein in rat arteriolar smooth muscle cells," Journal of Cellular Physiology, vol. 229, no. 12, pp. 1981-1989, 2014.

[40] R. Zhou, L. Liu, and D. Hu, "Involvement of BKCa alpha subunit tyrosine phosphorylation in vascular hyporesponsiveness of superior mesenteric artery following hemorrhagic shock in rats," Cardiovascular Research, vol. 68, no. 2, pp. 327-335, 2005.

[41] D. Y. Zhang, W. Wu, X. L. Deng, C. P. Lau, and G. R. Li, "Genistein and tyrphostin AG556 inhibit inwardly-rectifying Kir2.1 channels expressed in HEK 293 cells via protein tyrosine kinase inhibition," Biochimica et Biophysica Acta, vol. 1808, no. 8, pp. 1993-1999, 2011.

[42] D. Lin, E. J. Kamsteeg, Y. Zhang, et al., "Expression of tetraspan protein CD63 activates protein-tyrosine kinase (PTK) and enhances the PTK-induced inhibition of ROMK chan- nels," The Journal of Biological Chemistry, vol. 283, no. 12, pp. 7674-7681, 2008.

[43] J. Ross and W. M. Armstead, "Differential role of PTK and ERK MAPK in superoxide impairment of KATPand KCachannel cerebrovasodilation," American Journal of Physiology. Regulatory, Integrative and Comparative Physiology, vol. 285, no. 1, pp. R149-R154, 2003.

[44] S. Mergler, K. Steinhausen, M. Wiederholt, and O. Strauss, "Altered regulation of L-type channels by protein kinase $\mathrm{C}$ and protein tyrosine kinases as a pathophysiologic effect in retinal degeneration," The FASEB Journal, vol. 12, no. 12, pp. 1125-1134, 1998. 